\title{
GENERATING NEW CLASSES OF ORTHOGONAL POLYNOMIALS
}

\author{
AMILCAR BRANQUINHO \\ Departamento de Matemática \\ FCTUC \\ Universidade de Coimbra \\ Apartado 3008 \\ 3000 Coimbra \\ Portugal
}

\section{FRANCISCO MARCELLÁN}

Departamento de Ingeniería

Escuela Politécnica Superior

Universidad Carlos III

C. Butarque, 15

28911 Leganés-Madrid

Spain

(Received September 28, 1994 and in revised form December 15, 1994)

ABSTRACT. Given a sequence of monic orthogonal polynomials (MOPS), $\left\{P_{n}\right\}$, with respect to a quasi-definite linear functional $u$, we find necessary and sufficient conditions on the parameters $a_{n}$ and $b_{n}$ for the sequence

$$
\begin{aligned}
& P_{n}(x)+a_{n} P_{n-1}(x)+b_{n} P_{n-2}(x), n \geq 1 \\
& P_{0}(x)=1, P_{-1}(x)=0
\end{aligned}
$$

to be orthogonal. In particular, we can find explicitly the linear functional $v$ such that the new sequence is the corresponding family of orthogonal polynomials. Some applications for Hermite and Tchebychev orthogonal polynomials of second kind are obtained.

We also solve a problem of this type for orthogonal polynomials with respect to a Hermitian linear functional.

KEY WORDS: Orthogonal polynomials, Linear functionals, Quasi-orthogonality.

MATHEMATICS SUBJECT CLASSIFICATION (1991). Primary $33 C 45$ and Secondary $42 \mathrm{C} 05$.

\section{INTRODUCTION}

We consider two positive Borel measures $\mu_{1}, \mu_{2}$ supported on a set $I \subset \mathbb{R}$ with infinitely many points and such that $\mathrm{d} \mu_{2}=\frac{\mathrm{d} \mu_{1}}{q(x)}$ where $q(x)=\prod_{i=1}^{l}\left(x-x_{\imath}\right)$ has their zeros outside $I$. We denote by $\left\{P_{n}\right\}$ and $\left\{R_{n}\right\}$ the MOPS with respect to $\mu_{1}$ and $\mu_{2}$, respectively. Then, it is well known that

$$
R_{n}(x)=\frac{\left|\begin{array}{ccc}
P_{n}(x) & \ldots & P_{n-l}(x) \\
Q_{n}\left(x_{1}\right) & \ldots & Q_{n-l}\left(x_{1}\right) \\
& \ldots & \\
Q_{n}\left(x_{l}\right) & \ldots & Q_{n-l}\left(x_{l}\right)
\end{array}\right|}{\left|\begin{array}{ccc}
Q_{n-1}\left(x_{1}\right) & \ldots & Q_{n-l}\left(x_{1}\right) \\
Q_{n-1}\left(x_{l}\right) & \ldots & Q_{n-l}\left(x_{l}\right)
\end{array}\right|}
$$

where

$$
Q_{n}(s)=\int_{I} \frac{P_{n}(t)}{t-s} \mathrm{~d} \mu_{1}(t)
$$

(see [15] and [18]). This means that

$$
R_{n}(x)=P_{n}(x)+a_{n}^{(1)} P_{n-1}(x)+\ldots+a_{n}^{(l)} P_{n-l}(x)
$$

with $a_{n}^{(l)} \neq 0$.

We are interested in some partial converse of the above result when $l=2$ as well as some extensions for more general families of orthogonal polynomials. 
If $u_{1}$ and $u_{2}$ denote the linear functionals on the linear space $\mathbb{P}$ of polynomials with complex coefficients, defined respectively by

$$
\left\langle u_{\imath}, p(x)\right\rangle=\int_{I} p(x) \mathrm{d} \mu_{\imath}(x), \imath=1,2
$$

for every $p \in \mathbb{P}$, then

$$
\begin{aligned}
\left\langle q(x) u_{2}, p(x)\right\rangle & =\int_{I} q(x) p(x) \mathrm{d} \mu_{2}(x) \\
& =\int_{I} p(x) \mathrm{d} \mu_{1}(x)=\left\langle u_{1}, p(x)\right\rangle
\end{aligned}
$$

i.e. $q u_{2}=u_{1}$ in the space of linear functionals on $\mathbb{P}$.

If $q$ is a polynomial of degree two and $u_{1}$ is a quasi-definite linear functional our first problem is to find necessary and sufficient conditions in order to $u_{2}$ defined by $q u_{2}=u_{1}$ be a quasi-definite linear functional.

Maroni studied this problem when $p$ is a polynomial of degree 1 (see [13]) and in the case when $p$ is a polynomial of degree 2 with $x_{1}=x_{2}$ (see [14]). In this last case, he does not give the proof of this result. The conditions that we give here are basically different from that ones.

On the other hand, we consider the following inverse problem:

Given a MOPS $\left\{P_{n}\right\}$ with respect to a quasi-definite linear functional $u_{1}$, to characterize the sequences $\left(a_{n}\right),\left(b_{n}\right)$ of real numbers, such that the sequence of monic polynomials

$$
R_{n}=P_{n}+a_{n} P_{n-1}+b_{n} P_{n-2}, n \geq 1
$$

is orthogonal with respect to some quasi-definite linear functional $u_{2}$. As an immediate consequence, to find the relation between $u_{1}$ and $u_{2}$.

A first attempt to propose an inverse problem was made by J.L.Geronimus in [6]. In fact, he obtained information about the quasi-definite linear functionals $u$ and $v$ associated to two MOPS $\left\{P_{n}\right\}$ and $\left(R_{n}\right)$ related by (2). Furthermore, he gave necessary and sufficient conditions on the family $\left\{R_{n}\right\}$ in order to be orthogonal with respect to $v$.

This problem is also connected with positive quadrature formulae (see [19]).

Geronimus gave also in that paper a proof of the Hahn's theorem for the classical MOPS, i.e. the only MOPS $\left\{P_{n}\right\}$ such that the $\left\{\frac{P_{n+1}^{\prime}}{n+1}\right\}$ is also a MOPS are the classical ones. He used the fact that the classical MOPS satisfy

$$
\begin{aligned}
P_{n+2}(x) & =\frac{P_{n+3}^{\prime}}{n+3}(x)+a_{n+2} \frac{P_{n+2}^{\prime}}{n+2}(x)+b_{n+2} \frac{P_{n+1}^{\prime}}{n+1}(x), n \in \mathbb{N} \\
P_{1}(x) & =\frac{P_{2}^{\prime}}{2}(x)+a_{1} P_{1}^{\prime}(x), P_{0}(x)=1
\end{aligned}
$$

We have proved that this relation characterize classical MOPS (see [3]).

This problem is connected to another stated by Geronimus (see [7]):

- Construct a MOPS, $\left\{P_{n}\right\}$, which satisfy

$$
x P_{n+s+1}=P_{n+s+2}+\beta_{n+s+1} P_{n+s+1}+\gamma_{n+s} P_{n+s}, n \in \mathbb{N} .
$$

When $\left\{\begin{array}{l}\beta_{n+s+1}=0 \\ \gamma_{n+s}=\frac{1}{4}\end{array}, n \in \mathbb{N}\right.$ he gave a representation of the measure with respect to $\left\{P_{n}\right\}$ is orthogonal. This is because $\left\{P_{n}\right\}$ is a finite linear combination of second kind Tchebychev orthogonal polynomials.

Finally, we study an inverse problem for orthogonal polynomials with respect to Hermitian linear functionals on the linear space of Laurent polynomials. This represents a generalization of the theory of orthogonal polynomials with respect to a measure supported on the unit circle. 


\section{The structure of this work is the following:}

(a) In Section 2 we present the basic tools concerning linear functionals as well as the concept of quasi-orthogonality which will play a central role in our paper.

(b) Section 3 is devoted to the direct problem, i.e. the relation between orthogonal polynomials associated with two quasi-definite linear functionals $u, v$ satisfying a relation

$$
u=p(x) v
$$

where $\operatorname{deg} p=2$. Furthermore, we give in Theorem 3 the connection between the corresponding parameters of the three term recurrence relation.

(c) In Section 4 we study the inverse problem (3) and we consider some examples. Theorem 4.1 gives the characterization of such sequences by a constructive approach.

(d) In Section 5 we give necessary conditions on the sequence $a_{n}$ in order to

$$
\psi_{n}(z)=\phi_{n}(z)+a_{n} \phi_{n-1}(z)
$$

be a MOPS with respect to an Hermitian linear functional if $\left\{\phi_{n}\right\}$ is a MOPS with respect to an Hermitian linear functional.

\section{QUASI-ORTHOGONALITY}

We now introduce the algebraic tools that we use in this paper, (see [5] and [12] for more details). Let $\left\{P_{n}\right\}$ be a MOPS with respect to the quasi-definite (respectively, positive definite) linear functional $u$ defined on $\mathbb{P}$, i.e.

$$
\left\langle u, P_{n}(x) P_{m}(x)\right\rangle=k_{n} \delta_{n m} \text { with } k_{n} \neq 0 \text { (respectively, } k_{n} \geq 0 \text { ) for } n, m \in \mathbb{N},
$$

where $\langle.,$.$\rangle means the duality bracket. Furthermore, \left\{P_{n}\right\}$ satisfies the following three term recurrence relation

$$
\begin{aligned}
& x P_{n}(x)=P_{n+1}(x)+\beta_{n} P_{n}(x)+\gamma_{n} P_{n-1}(x) \text { for } n=1,2, \ldots \\
& P_{0}(x)=1, P_{1}(x)=x-\beta_{0} .
\end{aligned}
$$

where $\left(\beta_{n}\right)$ and $\left(\gamma_{n}\right)$ be two sequences of complex numbers with $\gamma_{n+1} \neq 0$ in the quasi-definite case and $\gamma_{n+1}>0,\left(\beta_{n}\right) \subset \mathbb{R}$ in the positive definite case, for $n \in \mathbb{N}$.

Since $\left\{P_{n}\right\}$ is an algebraic basis in $\mathbb{P}$, we can define its dual basis $\left(\alpha_{n}\right)$ in $\mathbb{P}^{*}$ (the algebraic dual space of $\mathbb{P})$ as $\left\langle\alpha_{n}, P_{m}\right\rangle=\delta_{m, n}$.

If $v$ is an element of $\mathbb{P}^{*}$, we can express it as $v=\sum_{\imath=0}^{\infty} \lambda_{\imath} \alpha_{\imath}$ where $\lambda_{\imath}=\left\langle v, P_{\imath}\right\rangle, i \in \mathbb{N}$. As an immediate consequence, if $v \in \mathbb{P}^{*}$ satisfies $\left\langle v, P_{\imath}\right\rangle=0$ for $i \geq l$, then $v=\sum_{i=0}^{l-1} \lambda_{\imath} \alpha_{1}$. In particular, $u=\mu_{0} \alpha_{0}$. We can represent the elements of the dual basis in terms of $\left\{P_{n}\right\}^{i=0}$ and $u$. In fact, since $\left\langle\alpha_{n}, P_{m}\right\rangle=\delta_{n, m}=\left\langle\frac{P_{n} u}{\left\langle u, P_{n}^{2}\right\rangle}, P_{m}\right\rangle, n, m \in \mathbb{N}$, we have

$$
\alpha_{n}=\frac{P_{n} u}{\left\langle u, P_{n}^{2}\right\rangle}, n \in \mathbb{N} .
$$

Let define some linear operators on $\mathbb{P}$ :

$$
\begin{aligned}
& p \mapsto(q p)(x)=q(x) p(x), q \in \mathbb{P} \\
& p \cdot \mapsto \quad\left(\theta_{c} p\right)(x)=\frac{p(x)-p(c)}{x-c}, c \in \mathbf{C}
\end{aligned}
$$

By transposition, we introduce the following linear operators on $\mathbb{P}^{*}$ :

1. $\langle q \alpha, p\rangle=\langle\alpha, q p\rangle$ where

$$
\left\langle q \alpha, x^{n}\right\rangle=\sum_{\imath=0}^{\operatorname{deg} q} a_{\imath}\left\langle\alpha, x^{\imath+n}\right\rangle, n \in \mathbb{N}
$$


for $q(x)=\sum_{i=0}^{\operatorname{deg} q} a_{i} x^{i}$ and $\alpha \in \mathbb{P}^{*}$

2. $\left\langle(x-c)^{-1} \alpha, p\right\rangle=\left\langle\alpha, \theta_{c} p\right\rangle$ where

$$
\left\langle(x-c)^{-1} \alpha, x^{n}\right\rangle=\left\{\begin{array}{l}
0, \text { if } n=0 \\
\sum_{i=0}^{n-1} c^{n-1-i}\left\langle\alpha, x^{i+n}\right\rangle, \text { if } n \geq 1
\end{array}\right.
$$

Given a polynomial $p(x)=\prod_{\imath=1}^{s}\left(x-x_{\imath}\right)^{m_{\imath}}$, for every $f \in \mathbb{P}$, we define

$$
\left(\Theta_{X} f\right)(x)=\frac{f(x)-(\mathcal{L} f)(x)}{p(x)}
$$

where,

$$
(\mathcal{L} f)(x)=\sum_{\imath=1}^{s} \sum_{k=1}^{m_{i}} f^{(k-1)}\left(x_{\imath}\right) L_{\imath k}(x)
$$

is the Lagrange-Sylvester (Hermite) interpolation polynomial of $f$ with nodes $x_{\imath}, i=1, \ldots, s$ and the $L_{i k}$ verify the following conditions:

$$
L_{\imath k}^{(\nu)}\left(x_{\jmath}\right)=\left\{\begin{array}{l}
1 \text { if } \nu=k-1 \text { and } j=i \\
0 \text { elsewhere }
\end{array}\right.
$$

for $k=1,2, \ldots, m_{\imath}$ and $i=1, \ldots, s$. Then:

DEFINITION $1 p^{-1} u$ is the linear functional given by

$$
\left\langle p^{-1}(x) u, f\right\rangle=\left\langle u, \Theta_{X} f\right\rangle .
$$

DEFINITION 2 ([5]) Let $\left\{P_{n}\right\}$ be a MOPS with respect to the quasi-definite linear functional $u$. We say $\left\{P_{n}^{(1)}\right\}$ is the first associated MOPS for the MOPS $\left\{P_{n}\right\}$ if it satisfies

$$
\begin{aligned}
& x P_{n}^{(1)}(x)=P_{n+1}^{(1)}(x)+\beta_{n+1} P_{n}^{(1)}(x)+\gamma_{n+1} P_{n-1}^{(1)}(x) \text { for } n=1,2, \ldots \\
& P_{0}^{(1)}(x)=1, P_{1}^{(1)}(x)=x-\beta_{1} .
\end{aligned}
$$

DEFINITION 3 ([4]) The MOPS defined by

$$
P_{n+1}(x ; c)=P_{n+1}(x)-c P_{n}^{(1)}(x), n \in \mathbb{N} .
$$

is called a co-recursive sequence of the MOPS $\left\{P_{n}\right\}$.

Notice that these polynomials verify the same three-term recurrence relation as $\left\{P_{n}\right\}$ with initial conditions $P_{0}(x ; c)=1$ and $P_{1}(x ; c)=P_{1}(x)-c$.

Next we introduce a basic definition in our work:

DEFINITION 4 ([14]) Let $\left\{p_{n}\right\}$ be a MPS with $\operatorname{deg} p_{n}=n$ and $u$ be a linear functional. We say that $\left\{p_{n}\right\}$ is strictly quasi-orthogonal of order $s$ if

$$
\begin{aligned}
& \left\langle u, p_{m} p_{n}\right\rangle=0,|n-m| \geq s+1 \\
& \forall r \geq s\left\langle u, p_{r-s} p_{r}\right\rangle \neq 0 .
\end{aligned}
$$

THEOREM 5 ([16]) Let $\left\{P_{n}\right\}$ be a MOPS with respect to the quasi-definite linear functional $u$; then, $\left\{p_{n}\right\}$ is strictly quasi-orthogonal of order $s$ with respect to $u$ if and only if

$$
p_{n}(x)=\left\{\begin{array}{l}
\sum_{k=0}^{n} a_{n, k} P_{k}, 0 \leq n \leq s-1 \\
\sum_{k=n-s+1}^{n} a_{n, k} P_{k}, n \geq s
\end{array} \quad \text { where } a_{n, n-s} \neq 0, \forall n \geq s .\right.
$$




\section{DIRECT PROBLEM}

This section is motivated by some modifications considered in the literature of orthogonal polynomials. As an example, the Bernstein-Szegö polynomials (see [5], [9] and [10]) are orthogonal with respect to the weight functions

$$
w(x)=\left\{\begin{array}{l}
\left(1-x^{2}\right)^{-1 / 2}(\rho(x))^{-1} \\
\left(1-x^{2}\right)^{1 / 2}(\rho(x))^{-1} \\
\left(\frac{1-x}{1+x}\right)^{1 / 2}(\rho(x))^{-1}
\end{array}\right.
$$

where $\rho$ is a positive polynomial in $[-1,1]$ of fixed degree. These polynomials can be represented in an easy way in terms of the Tchebychev Polynomials of first and second kind as can be deduced from (1) (see also [17, Theorem 2.6]).

Here we solve a more general problem:

THEOREM 6 ([2]) Let $u$ be a quasi-definite linear functional with $u_{0}=1$ and $x_{1} \neq x_{2} \in \mathbf{C}$. The linear functional $v$ defined by $u=\left(x-x_{1}\right)\left(x-x_{2}\right) v$ is quasi-definite if and only if $v_{0} \neq 0$ and $v_{1}$ are such that

$$
\begin{gathered}
\frac{x_{2} v_{0}-v_{1}}{x_{2}-x_{1}}\left(x_{1}-v_{1}\right)^{2}+\frac{v_{1}-x_{1} v_{0}}{x_{2}-x_{1}}\left(x_{2}-v_{1}\right)^{2} \neq 1 \\
\left|d_{n}\right| \neq 0, n \in \mathbb{N}
\end{gathered}
$$

Here

$$
d_{n}=\left[\begin{array}{ll}
P_{n+1}\left(x_{1} ;-\frac{1}{v_{1}-x_{2} v_{0}}\right) & P_{n}\left(x_{1} ;-\frac{1}{v_{1}-x_{2} v_{0}}\right) \\
P_{n+1}\left(x_{2} ;-\frac{1}{v_{1}-x_{1} v_{0}}\right) & P_{n}\left(x_{2} ;-\frac{1}{v_{1}-x_{1} v_{0}}\right)
\end{array}\right]
$$

and $v_{0}, v_{1}$ are the first and second moments of $v$, respectively. In this situation the MOPS with respect to $v$ is given by

$$
\begin{aligned}
& R_{n+2}(x)=P_{n+2}(x)+a_{n+2} P_{n+1}(x)+b_{n+2} P_{n}(x), n \in \mathbb{N} \\
& R_{1}(x)=P_{1}(x)+a_{1} P_{0}(x) \\
& R_{0}(x)=P_{0}(x)
\end{aligned}
$$

where

$$
\left[\begin{array}{c}
a_{n+2} \\
b_{n+2}
\end{array}\right]=-d_{n}^{-1}\left[\begin{array}{l}
P_{n+2}\left(x_{1} ;-\frac{1}{v_{1}-x_{2} v_{0}}\right) \\
P_{n+2}\left(x_{2} ;-\frac{1}{v_{1}-x_{1} v_{0}}\right)
\end{array}\right], n \in \mathbb{N}
$$

and $a_{1}=\beta_{0}-v_{1}$. The coefficients of the corresponding recurrence relation

$$
\begin{aligned}
& x R_{n+1}(x)=R_{n+2}(x)+\xi_{n+1} R_{n+1}(x)+\eta_{n+1} R_{n}(x), n \in \mathbb{N} \\
& R_{1}(x)=x-\xi_{0}, R_{0}(x)=1
\end{aligned}
$$

are given by

$$
\left.\begin{array}{c}
\xi_{n}=\beta_{n}-\left(a_{n+1}-a_{n}\right), n \in \mathbb{N} \\
\eta_{n+3}=\frac{b_{n+3}}{b_{n+2}} \gamma_{n+1}, n \in \mathbb{N} \\
\eta_{1}=\gamma_{1}+a_{1}\left(\beta_{0}-\xi_{1}\right)-b_{2} \\
\eta_{2}=\gamma_{2}+a_{2}\left(\beta_{1}-\xi_{2}\right)-\left(b_{3}-b_{2}\right)
\end{array}\right\}
$$

LEMMA 7 Let $u, v$ be the linear functionals defined in the above theorem; then

$$
v=\left(x-x_{1}\right)^{-1}\left(x-x_{2}\right)^{-1} u+\frac{x_{2} v_{0}-v_{1}}{x_{2}-x_{1}} \delta_{x_{1}}+\frac{v_{1}-x_{1} v_{0}}{x_{2}-x_{1}} \delta_{x_{2}}
$$

where $\delta_{x_{\mathrm{t}}}$ means the Dirac mass at $x_{\mathfrak{\imath}}$.

PROOF OF THE LEMMA. We only need to determine

$$
\left(x-x_{1}\right)^{-1}\left(x-x_{2}\right)^{-1}\left(\left(x-x_{1}\right)\left(x-x_{2}\right) v\right)
$$




$$
\begin{aligned}
\left\langle\left(x-x_{1}\right)^{-1}\left(x-x_{2}\right)^{-1}\left(\left(x-x_{1}\right)\left(x-x_{2}\right) v\right), f(x)\right\rangle \\
=\left\langle\left(x-x_{1}\right)\left(x-x_{2}\right) v, \frac{\frac{f(x)-f\left(x_{1}\right)}{x-x_{1}}-\frac{f\left(x_{2}\right)-f\left(x_{1}\right)}{x_{2}-x_{1}}}{x-x_{2}}\right\rangle \\
=\left\langle v, f(x)+\frac{x-x_{2}}{x_{2}-x_{1}} f\left(x_{1}\right)-\frac{x-x_{1}}{x_{2}-x_{1}} f\left(x_{2}\right)\right\rangle \\
=\left\langle v+\frac{v_{1}-x_{2} v_{0}}{x_{2}-x_{1}} \delta_{x_{1}}+\frac{x_{1} v_{0}-v_{1}}{x_{2}-x_{1}} \delta_{x_{2}}, f(x)\right\rangle ;
\end{aligned}
$$

so, we get $(24)$.

PROOF OF THE THEOREM. From the above lemma, it seems natural that the necessary and sufficient conditions for the quasi-difiniteness of $v$ were given in terms of $x_{1}, x_{2}, v_{0}$ and $v_{1}$.

As $\left\{P_{n}\right\}$ is a basis of $\mathbb{P}$, we have $R_{n+2}(x)=P_{n+2}(x)+\sum_{k=0}^{n+1} a_{n, k} P_{k}(x)$, where the coefficients $a_{n, k}$ can be obtained as follows:

$$
\begin{aligned}
\left\langle u, P_{k}^{2}\right\rangle a_{n, k} & =\left\langle v,\left(x-x_{1}\right)\left(x-x_{2}\right) R_{n+2} P_{k}\right\rangle \\
& =\left\{\begin{array}{cl}
0 & , \text { if } k=0,1, \ldots, n-1 \\
\left\langle v, R_{n+2}^{2}\right\rangle & , \text { if } k=n \\
\left\langle v,\left(x-x_{1}\right)\left(x-x_{2}\right) R_{n+2} P_{n+1}\right\rangle & , \text { if } k=n+1
\end{array}\right.
\end{aligned}
$$

and so,

$$
R_{n+2}(x)=P_{n+2}(x)+a_{n+2} P_{n+1}(x)+b_{n+2} P_{n}(x),
$$

where $a_{n+2}=a_{n, n+1}$ and $b_{n+2}=a_{n, n}$; so, a necessary condition for the quasi-definitnes of the linear functional $v$ is that $b_{n+2} \neq 0$ for $n \in \mathbb{N}$ (by (5)).

Taking into account theorem 5 , the MOPS with respect to $v$ is a strictly quasi-orthogonal MPS of order two with respect to $u$. Now, if we substitute in definition $4 u$ by $\left(x-x_{1}\right)\left(x-x_{2}\right) v$ we obtain that $v$ is quasi-definite (because $v$ is strictly quasi-orthogonal of order two with respect to $u$, i.e $\left.b_{n+2} \neq 0, n \in \mathbb{N}\right)$, if and only if:

i.1 $\left\langle v, R_{n+1}\right\rangle=0$ for $n \in \mathbb{N}$,

i. $2\left\langle v, x R_{n+2}\right\rangle=0$ for $n \in \mathbb{N}$,

i.3 $\left\langle v, R_{1}^{2}\right\rangle \neq 0$.

Expressing these conditions in terms of the data:

1. From i.1 with $n=0$ we obtain $a_{1}=\beta_{0}-v_{1}$.

2. From i.3 and taking into account the last expression for $a_{1}$ we get $\frac{x_{2} v_{0}-v_{1}}{x_{2}-x_{1}}\left(x_{1}-v_{1}\right)^{2}+$ $\frac{v_{1}-x_{1} v_{0}}{x_{2}-x_{1}}\left(x_{2}-v_{1}\right)^{2} \neq 1$.

3. From $\left\{\begin{array}{l}\left\langle v, R_{n+2}\right\rangle=0 \\ \left\langle v, x R_{n+2}\right\rangle=0\end{array}, n \in \mathbb{N}\right.$ we conclude that $\left\{\begin{array}{l}\left\langle v,\left(x-x_{1}\right) R_{n+2}\right\rangle=0 \\ \left\langle v,\left(x-x_{2}\right) R_{n+2}\right\rangle=0\end{array}\right.$ for $n \in \mathbb{N}$.

These two expressions are enough in order to determine $a_{n+2}$ and $b_{n+2}$ :

- Substituting $x$ by $x_{1}$ in (25) and substracting from (25) the equation we have found, we get, after dividing by $x-x_{1}$

$$
\theta_{x_{1}} R_{n+2}(x)=\theta_{x_{1}} P_{n+2}(x)+a_{n+2} \theta_{x_{1}} P_{n+1}(x)+b_{n+2} \theta_{x_{1}} P_{n}(x) .
$$

Applying the quasi-definite functional $u$ to both members of (26) and so on, we get

$$
\left\langle v,\left(x-x_{2}\right)\left(R_{n+2}(x)-R_{n+2}\left(x_{1}\right)\right)\right\rangle=P_{n+1}^{(1)}\left(x_{1}\right)+a_{n+2} P_{n}^{(1)}\left(x_{1}\right)+b_{n+2} P_{n-1}^{(1)}\left(x_{1}\right), n \in \mathbb{N} ;
$$

but we know that $\left\langle v,\left(x-x_{2}\right) R_{n+2}(x)\right\rangle=0$, so

$$
\left(v_{1}-x_{2} v_{0}\right) R_{n+2}\left(x_{1}\right)=P_{n+1}^{(1)}\left(x_{1}\right)+a_{n+2} P_{n}^{(1)}\left(x_{1}\right)+b_{n+2} P_{n-1}^{(1)}\left(x_{1}\right), n \in \mathbb{N} \text {. }
$$

- Using the same process described above with $x_{2}$ instead of $x_{1}$ we get

$$
\left(v_{1}-x_{1} v_{0}\right) R_{n+2}\left(x_{2}\right)=P_{n+1}^{(1)}\left(x_{2}\right)+a_{n+2} P_{n}^{(1)}\left(x_{2}\right)+b_{n+2} P_{n-1}^{(1)}\left(x_{2}\right), n \in \mathbb{N}
$$


and taking into account (25) we obtain

$$
\left\{\begin{array}{l}
P_{n+1}\left(x_{1} ;-\frac{1}{v_{1}-x_{2} v_{0}}\right) a_{n+2}+P_{n}\left(x_{1} ;-\frac{1}{v_{1}-x_{2} v_{0}}\right) b_{n+2}=-P_{n+2}\left(x_{1} ;-\frac{1}{v_{1}-x_{2} v_{0}}\right) \\
P_{n+1}\left(x_{2} ;-\frac{1}{v_{1}-x_{1} v_{0}}\right) a_{n+2}+P_{n}\left(x_{2} ;-\frac{1}{v_{1}-x_{1} v_{0}}\right) b_{n+2}=-P_{n+2}\left(x_{2} ;-\frac{1}{v_{1}-x_{1} v_{0}}\right)
\end{array}\right.
$$

and from this, we get $(20)$. Hence

$$
b_{n+2}=-\frac{\left|d_{n+1}\right|}{\left|d_{n}\right|}, n \in \mathbb{N}
$$

As a conclusion:

- The linear functional $v$ is quasi-definite if and only if (18) is verified.

Concerning the determination of the coefficients of the recurrence relation (21):

Substituting $R_{n+1}$ in $(21)$ by $P_{n+1}(x)+a_{n+1} P_{n}(x)+b_{n+1} P_{n-1}(x)$ and applying the recurrence relation (6) we get, after comparing the coefficients of the $P_{k}$ with $k=n-2, n-1, n, n+1$,

(a) $\eta_{n+1}=\frac{b_{n+1}}{b_{n}} \gamma_{n-1}, n \geq 2$

(b) $a_{n+1} \gamma_{n}+b_{n+1} \beta_{n-1}=\xi_{n+1} b_{n+1}+\eta_{n+1} a_{n}, n \geq 1$

(c) $\gamma_{n+1}+a_{n+1} \beta_{n}+b_{n+1}=b_{n+2}+\xi_{n+1} a_{n+1}+\eta_{n+1}, n \geq 0$

(d) $\xi_{n+1}=\beta_{n+1}-\left(a_{n+2}-a_{n+1}\right), n \geq-1$

As a limit case we have:

COROLLARY 8 ([2],[14]) Let $u$ be a quasi-definite linear functional with $u_{0}=1$ and $x_{1} \in$ $\mathbf{C}$; the linear functional $v$ defined by $u=\left(x-x_{1}\right)^{2} v$ is quasi-definite, if and only if $v_{0} \neq 0$ and $v_{1}$ are such that

$$
\begin{aligned}
& \frac{\left(v_{1}-x_{1} v_{0}\right)^{2}}{v_{0}} \neq 1 \\
& \left|d_{n}\right|^{\neq} \neq 0, \text { for every } n \in \mathbb{N}
\end{aligned}
$$

where $d_{n}$ is the matrix

$$
\left[\begin{array}{cc}
P_{n+1}\left(x_{1} ;-\frac{1}{v_{1}-x_{1} v_{0}}\right) & P_{n}\left(x_{1} ;-\frac{1}{v_{1}-x_{1} v_{0}}\right) \\
\left(v_{1}-x_{1} v_{0}\right) P_{n+1}^{\prime}\left(x_{1} ;-\frac{1}{v_{1}-x_{1} v_{0}}\right)+v_{0} P_{n+1}\left(x_{1}\right) & \left(v_{1}-x_{1} v_{0}\right) P_{n}^{\prime}\left(x_{1} ;-\frac{1}{v_{1}-x_{1} v_{0}}\right)+v_{n}\left(x_{1}\right)
\end{array}\right] .
$$

The corresponding MOPS with respect to $v$ is given by

$$
R_{n+2}(x)=P_{n+2}(x)+a_{n+2} P_{n+1}(x)+b_{n+2} P_{n}(x), n \in \mathbb{N},
$$

where

$$
\left[\begin{array}{l}
a_{n+2} \\
b_{n+2}
\end{array}\right]=-d_{n}^{-1}\left[\begin{array}{c}
P_{n+2}\left(x_{1} ;-\frac{1}{v_{1}-x_{1} v_{0}}\right) \\
\left(v_{1}-x_{1} v_{0}\right) P_{n+2}^{\prime}\left(x_{1} ;-\frac{1}{v_{1}-x_{1} v_{0}}\right)+v_{0} P_{n+2}\left(x_{1}\right)
\end{array}\right]
$$

and $a_{1}=\beta_{0}-\frac{v_{1}}{v_{0}}$. The coefficients of the recurrence relation that $R_{n}$ satisfies (21) are related with the ones of (6) by the following formulas

$$
\left.\begin{array}{c}
\xi_{n}=\beta_{n}-\left(a_{n+1}-a_{n}\right), n \in \mathbb{N} \\
\eta_{n+3}=\frac{b_{n+3}}{b_{n+2}} \gamma_{n+1}, n \in \mathbb{N} \\
\eta_{1}=\gamma_{1}+a_{1}\left(\beta_{0}-\xi_{1}\right)-b_{2} \\
\eta_{2}=\gamma_{2}+a_{2}\left(\beta_{1}-\xi_{2}\right)-\left(b_{3}-b_{2}\right)
\end{array}\right\}
$$

\section{REAL INVERSE PROBLEM}

The answer to the main problem is the following: 
THEOREM 9 Let $\left\{P_{n}\right\}$ be a MOPS with respect to u and $\left\{R_{n}\right\}$ be a strictly quasi-orthogonal MPS of order two with respect to $\left\{P_{n}\right\} ;\left\{R_{n}\right\}$ is a MOPS if and only if, the parameters in (16) $a_{n, n}=a_{n}$ and $a_{n, n-1}=b_{n}$ satisfy

$$
\begin{aligned}
& a_{n+4}=\frac{a_{2}}{b_{2}} \gamma_{1}-\beta_{2}-\beta_{3}+a_{3}-\frac{a_{n+3}}{b_{n+3}} \gamma_{n+2}+\beta_{n+3}+\beta_{n+2} \\
& b_{n+4}=b_{n+3}\left(1-\frac{\gamma_{n+1}}{b_{n+2}}\right)+\gamma_{n+3}+a_{n+3}\left(\beta_{n+2}-\beta_{n+3}+a_{n+4}-a_{n+3}\right)
\end{aligned}, n \in \mathbb{N}
$$

where the inıtial conditions are

(a) $a_{1}, a_{2}, b_{2}, b_{3}$ if

$$
a_{1}=0 \text { and } a_{3}=\beta_{2}-\beta_{0}+a_{2}-\frac{a_{2}}{b_{2}} \gamma_{1}
$$

$$
a_{1} \neq 0, b_{2}-a_{1} a_{2} \neq 0 \text { and } a_{3}=\frac{a_{2} \gamma_{1}+b_{2}\left(\beta_{0}-\beta_{2}-a_{2}\right)-a_{1}\left(\gamma_{2}+a_{2}\left(\beta_{1}-\beta_{2}-a_{2}\right)-b_{3}+b_{2}\right)}{a_{1} a_{2}-b_{2}}
$$

(b) $a_{1}, a_{2}, a_{3}, b_{3}$ if $a_{1} \neq 0$ and $b_{2}=a_{1} a_{2}$.

Furthermore, $a_{1}, a_{2}, a_{3}, b_{2}, b_{3}$ verify

$$
\left.\begin{array}{r}
\gamma_{2}+a_{2}\left(\beta_{1}-\beta_{2}+a_{3}-a_{2}\right)-b_{3}+b_{2} \neq 0 \\
\gamma_{1}+a_{1}\left(\beta_{0}-\beta_{1}+a_{2}-a_{1}\right)-b_{2} \neq 0
\end{array}\right\}
$$

In this case, the coefficients of the recurrence relation that $\left\{R_{n}\right\}$ satisfies are given by

$$
\left\{\begin{array}{l}
\xi_{n}=\beta_{n}-\left(a_{n+1}-a_{n}\right) \\
\eta_{n+1}=\gamma_{n+1}+a_{n+1}\left(\beta_{n}-\xi_{n+1}\right)-\left(b_{n+2}-b_{n+1}\right)
\end{array}, n \in \mathbb{N}\right.
$$

with the restrictions $a_{0}=b_{1}=0$.

In addition, the linear functional $v$ such that $\left\{R_{n}\right\}$ is the corresponding MOPS is given by

$$
u=p(x) v,
$$

where $p(x)=\frac{1}{\langle v, 1\rangle}\left[\left(1+\frac{a_{1}^{2}}{\eta_{1}}+\frac{b_{2}^{2}}{\eta_{1} \eta_{2}}\right) P_{0}(x)+\left(\frac{a_{1}}{\eta_{1}}+\frac{a_{2} b_{2}}{\eta_{1} \eta_{2}}\right) P_{1}(x)+\frac{b_{2}}{\eta_{1} \eta_{2}} P_{2}(x)\right]$.

\section{REMARKS:}

1. From (38) and taking into account theorem 6 and its corollary we have determined $a_{n}$ and $b_{n}$ :

- If $\left(\frac{a_{1} \eta_{2}}{b_{2}}-a_{2}\right)^{2}-4\left(\frac{\eta_{1} \eta_{2}}{b_{2}}+\frac{a_{1}^{2} \eta_{2}}{b_{2}}+b_{2}-\gamma_{1}\right)=0$ then (33) appears.

- If $\left(\frac{a_{1} \eta_{2}}{b_{2}}-a_{2}\right)^{2}-4\left(\frac{\eta_{1} \eta_{2}}{b_{2}}+\frac{a_{1}^{2} \eta_{2}}{b_{2}}+b_{2}-\gamma_{1}\right) \neq 0$ then (20) holds.

2. (36) provides an algorithm for the computation of $\left(a_{n}, b_{n}\right)$. In fact, from the first relation we can calculate the $a_{n+4}$ in terms of $\left(a_{k}\right)_{k=2}^{n+3}$ and $\left(b_{k+1}\right)_{k=1}^{n+2}$; and substituting $a_{n+4}$ in the second one we get $b_{n+4}$.

PROOF. From theorem 5, there exist two sequences $\left(a_{n}\right),\left(b_{n}\right) \subset \mathbb{R}$ such that

$$
R_{n+2}(x)=P_{n+2}(x)+a_{n+2} P_{n+1}(x)+b_{n+2} P_{n}(x)
$$

with $b_{n+2} \neq 0, n \in \mathbb{N}$. Multiplying the equation (39) by $x$ and using (6) we obtain

$$
\begin{array}{r}
x R_{n+2}=P_{n+3}+\beta_{n+2} P_{n+2}+\gamma_{n+2} P_{n+1}+a_{n+2}\left(P_{n+2}+\beta_{n+1} P_{n+1}+\gamma_{n+1} P_{n}\right)+ \\
b_{n+2}\left(P_{n+1}+\beta_{n} P_{n}+\gamma_{n} P_{n-1}\right)
\end{array}
$$


Applying two times (39) we have

$$
\begin{aligned}
x R_{n+2}= & R_{n+3}+\xi_{n+2} R_{n+2}+\eta_{n+2} R_{n+1}+ \\
& \left(a_{n+2} \gamma_{n+1}-a_{n+1} \eta_{n+2}+b_{n+2}\left(\beta_{n}-\xi_{n+2}\right)\right) P_{n}+\left(b_{n+2} \gamma_{n}-b_{n+1} \eta_{n+2}\right) P_{n-1}
\end{aligned}
$$

where

$$
\left\{\begin{array}{l}
\xi_{n}=\beta_{n}-\left(a_{n+1}-a_{n}\right) \\
\eta_{n+1}=\gamma_{n+1}+a_{n+1}\left(\beta_{n}-\xi_{n+1}\right)-\left(b_{n+2}-b_{n+1}\right)
\end{array}, n \in \mathbb{N} .\right.
$$

So, $\left\{R_{n}\right\}$ is a MOPS if and only if

$$
\left\{\begin{array}{l}
\gamma_{n+1}+a_{n+1}\left(\beta_{n}-\xi_{n+1}\right)-\left(b_{n+2}-b_{n+1}\right) \neq 0 \\
a_{n+2} \gamma_{n+1}-a_{n+1} \eta_{n+2}+b_{n+2}\left(\beta_{n}-\xi_{n+2}\right)=0, n \in \mathbb{N} \\
b_{n+3} \gamma_{n+1}-b_{n+2} \eta_{n+3}=0
\end{array}\right.
$$

Now if we substitute $\eta_{n+3}=\frac{b_{n+3} \gamma_{n+1}}{b_{n+2}}$ in the second equation of (41) and in the expression of $\eta_{n+1}$ of (40) we get

$$
\begin{aligned}
\frac{a_{n+3}}{b_{n+3}} \gamma_{n+2}-\frac{a_{n+2}}{b_{n+2}} \gamma_{n+1}+\left(a_{n+4}-a_{n+3}\right)-\left(\beta_{n+3}-\beta_{n+1}\right) & =0 \\
\gamma_{n+3}+a_{n+3}\left(\beta_{n+2}-\beta_{n+3}+a_{n+4}-a_{n+3}\right)-\left(b_{n+4}-b_{n+3}\right) & =\frac{b_{n+3} \gamma_{n+1}}{b_{n+2}}
\end{aligned}
$$

and from this, the algorithm (36) follows.

Now $\left\{R_{n}\right\}$ is a MOPS associated with the quasi-definite functional $v$. We search for a relation between these two functionals:

- If we apply the functional $u$ in (39) we obtain $\left\langle u, R_{n+2}\right\rangle=0, n \in \mathbb{N}$. Hence

$$
u=\sum_{i=0}^{2} \lambda_{\imath} \alpha_{i}
$$

where $\left(\alpha_{n}\right)$ is the dual basis associated with $\left\{R_{n}\right\}$ and $\lambda_{\imath}=\left\langle u, R_{\imath}\right\rangle, i=0,1,2$.

As $\left\{R_{n}\right\}$ is a MOPS associated with $v$ we have by $(7) \alpha_{n}=\frac{R_{n}}{\left\langle v, R_{n}^{2}\right\rangle} v$ for all $n \in \mathbb{N}$; and so (42) can be rewritten as

$$
u=\left(1+\frac{a_{1}}{\eta_{1}} R_{1}(x)+\frac{b_{2}}{\eta_{1} \eta_{2}} R_{2}(x)\right) \frac{v}{\langle v, 1\rangle} .
$$

Taking into account $R_{n}$ for $n=1,2$ we get $(38)$.

EXAMPLES. We will construct two MOPS strictly quasi-orthogonal of order 2 with respect to:

(1) Second kind Tchebychev MOPS, $\left\{U_{n}\right\}$.

It is well known that in this case $\left\{\begin{array}{l}\beta_{n}=0 \\ \gamma_{n+1}=\frac{1}{4}\end{array}, n \in \mathbb{N}\right.$ (see [5, Exercise 4.9]).

Hence, if we choose as initial conditions $a_{1}=a_{2}=2 a$ and $b_{2}=b_{3}=-c \neq 0$, with $a, c \in \mathbf{C}$, we obtain by induction from (36) and considerations about initial conditions, $\left\{\begin{array}{l}a_{n+3}=2 a \\ b_{n+4}=-c\end{array}, n \in \mathbb{N}\right.$.

It is straightforward to prove that (37) holds.

The coefficients of the three term recurrence relation (ttrr) verified by $\left\{R_{n}\right\}$ are

$$
\left\{\begin{array} { l } 
{ \xi _ { 0 } = - 2 a } \\
{ \xi _ { n + 1 } = 0 }
\end{array} \text { and } \left\{\begin{array}{l}
\eta_{1}=\frac{1}{4}+c \\
\eta_{n+2}=\frac{1}{4}
\end{array}, n \in \mathbb{N}\right.\right.
$$


This is an example of Al-Salam and A. Verma (see [1]). There they found a measure such that $\left\{R_{n}\right\}$ is the corresponding MOPS. We can say that the condition for the positive definitness of the new linear functional is $c>-\frac{1}{4}$ and $a \in \mathbb{R}$.

Now, we give the quasi-definite (not positive definite, because $\eta_{1}<0$ ) linear functional, $\mathrm{v}$, in terms of the second kind Tchebychev linear functional, $u$, in the particular case $a=0$ and $c=-1$ :

$$
-\frac{16}{3 v_{0}} v=\left(\frac{9}{16}+x^{2}\right)^{-1} u+\frac{v_{0}}{2}\left(\delta_{-\frac{3}{4}}+\delta_{\frac{3}{4}}\right) .
$$

From this representation we get $\left\langle-\frac{16}{3 v_{0}} v, 1\right\rangle=v_{0}$, i.e $v_{0}=-\frac{16}{3}$; hence

$$
v=\left(\frac{9}{16}+x^{2}\right)^{-1} u-\frac{8}{3}\left(\delta_{-\frac{3}{4} 2}+\delta_{\frac{3}{4}}\right) .
$$

(2) Hermite MOPS, $\left\{H_{n}\right\}$.

It is well known that $\left\{\begin{array}{l}\beta_{n}=0 \\ \gamma_{n+1}=\frac{n+1}{2}\end{array}, n \in \mathbb{N}\right.$ (see [5, Exercise 1.6]).

Hence, if we choose as initial conditions $a_{1}=a_{2}=0$ and $b_{2}=b_{3}=1$ we obtain from (36) and

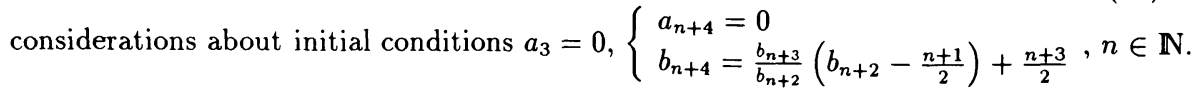

It can be seen $b_{2 k+2}=b_{2 k+3}=k+1$ for $k \in \mathbb{N}$. In fact,

$k=0 \quad b_{2}=b_{3}=1$. Using induction,

$$
\text { if } b_{2 k+2}=b_{2 k+3}=k+1 \text { for } k \leq p
$$

then $b_{2 k+2}=b_{2 k+3}=k+1$ for $k=p+1$. In fact

and

$$
b_{2 p+4}=\frac{2 p+3}{2}+\frac{p+1}{p+1}\left(p+1-\frac{2 p+1}{2}\right)=p+2
$$

$$
b_{2 p+5}=\frac{2 p+4}{2}+\frac{p+2}{p+1}\left(p+1-\frac{2 p+2}{2}\right)=p+2 .
$$

(37) can be deduced easily.

The coefficients of the ttrr verified by $\left\{R_{n}\right\}$ are given by

$$
\xi_{n}=0 \text { and } \eta_{n+1}=\left\{\begin{array}{l}
-\frac{1}{2} \text { if } n=0 \\
k+1 \text { if } n=2 k+1, n \in \mathbb{N} . \\
\frac{2 k+1}{2} \text { if } n=2 k+2
\end{array}\right.
$$

The quasi-definite (not positive definite) functional $v$ is given in terms of the Hermite linear functional, $u$, by:

$$
-\frac{2}{v_{0}} v=x^{-2} u+v_{0} \delta_{0}
$$

From this representation we get $\left\langle-\frac{2}{v_{0}} v, 1\right\rangle=v_{0}$, i.e $v_{0}=-2$; hence

$$
v=x^{-2} u-2 \delta_{0}
$$

\section{COMPLEX INVERSE PROBLEM}

Before working out the problem (4) we will state some basic definitions.

Let $\mathcal{M}=\left[\begin{array}{ccc}c_{0} & c_{1} & \ldots \\ \bar{c}_{1} & c_{0} & \cdots \\ & \cdots & \end{array}\right]$ be an infinite Toeplitz and Hermitian matrix. A linear functional $u$ on the linear space of Laurent polynomials $\mathcal{L}=\operatorname{span}\left\{z^{n}: n \in \mathbb{Z}\right\}$ is said to be Hermitian if $c_{n}=\left\langle u, z^{n}\right\rangle, n \in \mathbb{N}$ and $\bar{c}_{n}=\left\langle u, z^{-n}\right\rangle, n \in \mathbb{N}$. Here $\mathbb{Z}$ denotes the set of integers $\{0, \pm 1, \pm 2, \ldots\}$. The linear functional $u$ is said to be quasi-definite when the principal submatrices of $\mathcal{M}$ are non-singular. 
THEOREM $10([8]) \mathcal{M}$ represents the Gram matrix for the generalized inner product:

$$
\langle p(z), q(z)\rangle=\left\langle u, p(z) \bar{q}\left(\frac{1}{z}\right)\right\rangle
$$

associated with $u$ in $\mathbb{P}$.

In these conditions, we can define a MOPS $\left\{\phi_{n}\right\}$ with respect to the matrix $\mathcal{M}$ or with respect to the quasi-definite Hermitian linear functional $u$ by

$$
\phi_{n}(z)=\frac{1}{\Delta_{n-1}}\left|\begin{array}{cccc}
c_{0} & c_{1} & \ldots & c_{n} \\
\bar{c}_{1} & c_{0} & \ldots & c_{n-1} \\
& \ldots & & \\
\bar{c}_{n-1} & \bar{c}_{n-2} & \ldots & c_{1} \\
1 & z & \ldots & z^{n}
\end{array}\right|
$$

where $\Delta_{n-1}$ means the principal minor of order $n$ for $\mathcal{M}$. This family will be called a MOPS on the unit circle $T$ since the shift operator on $\mathbb{P}$ is unitary with respect to the above inner product.

Now, if we denote $K_{n}=\frac{\Delta_{n}}{\Delta_{n-1}}$ for $n \geq 1$ and $K_{0}=c_{0}$, it can be seen that $\frac{K_{n}}{K_{n-1}}=1-\left|\phi_{n}(0)\right|^{2}$; so $\left|\phi_{n}(0)\right| \neq 1$ for $n \geq 1$.

For a MOPS $\left\{\phi_{n}\right\}$ on the unit circle, $T$, Szegö recurrence relations follows (see [17, T. 11.4.2]), i.e.

$$
\begin{aligned}
& \phi_{n+1}(z)=z \phi_{n}(z)+\phi_{n+1}(0) \phi_{n}^{\star}(z) \\
& \phi_{n+1}^{\star}(z)=\phi_{n}^{\star}(z)+\overline{\phi_{n+1}(0)} z \phi_{n}(z)
\end{aligned}
$$

where $\left|\phi_{n+1}(0)\right| \neq 1$ and $\phi_{n}^{\star}(z)=z^{n} \overline{\phi_{n}}\left(z^{-1}\right)$ for $n \in \mathbb{N}$. Conversely, if $\left|\phi_{n+1}(0)\right| \neq 1, n \in \mathbb{N}$ the sequence defined by (44) is orthogonal with respect to a quasi-definite Hermitian linear functional (see [8]). The values $\left(\phi_{n}(0)\right)$ are called reflection parameters.

We define a MPS $\left\{\psi_{n}\right\}$ by

$$
\psi_{n}(z)=\phi_{n}(z)+a_{n} \phi_{n-1}(z) \text { for } n \geq 1 .
$$

But, $\left\{\psi_{n}\right\}$ is a MOPS on the unit circle if and only if verifies the following recurrence relation

$$
\psi_{n+1}(z)=z \psi_{n}(z)+\psi_{n+1}(0) \psi_{n}^{\star}(z), n \in \mathbb{N} \text { and }\left|\psi_{n+1}(0)\right| \neq 1 .
$$

So, using (46) we get

$$
\phi_{n+1}(z)+a_{n+1} \phi_{n}(z)=z \phi_{n}(z)+a_{n} z \phi_{n-1}(z)+\left(\phi_{n+1}(0)+a_{n+1} \phi_{n}(0)\right)\left(\phi_{n}^{\star}(z)+\bar{a}_{n} z \phi_{n-1}^{\star}(z)\right)
$$

for $n \geq 1$. Applying two times (44) we obtain

$$
\left[a_{n+1}-a_{n}-a_{n+1}\left|\phi_{n}(0)\right|^{2}\right] z \phi_{n-1}(z)=\left[\phi_{n+1}(0)+a_{n+1} \phi_{n}(0)\right] \bar{a}_{n} z \phi_{n-1}^{\star}(z)
$$

for $n \geq 1$. We can state the following result,

THEOREM 11 Let $\left\{\psi_{n}\right\}$ be a MPS defined by (46). If $\left\{\psi_{n}\right\}$ is orthogonal with respect to a Hermitian linear functional u then $\psi_{n}(z)=z^{n-2} \psi_{2}(z), n \geq 2$, where $\psi_{2}$ is a polynomial of degree two.

PROOF. From (47) and taking into account [11, proposition 2.3 (c)] we have

$$
\begin{aligned}
a_{2}\left(1-\left|\phi_{1}(0)\right|^{2}\right) & =\left(\phi_{2}(0)+a_{2} \phi_{1}(0)\right) \bar{a}_{1}+a_{1} \\
\bar{a}_{n}\left(\phi_{n+1}(0)+a_{n+1} \phi_{n}(0)\right) & =\bar{a}_{n} \psi_{n+1}(0)=0, n \geq 2 \\
a_{n+1}\left(1-\left|\phi_{n}(0)\right|^{2}\right) & =\frac{K_{n}}{K_{n-1}}=a_{n}, n \geq 2 .
\end{aligned}
$$

Now we consider three cases:

(a) If $a_{1}=0 \stackrel{(48)}{\Longrightarrow} a_{2}=0 \stackrel{(50)}{\Longrightarrow} a_{n}=0, n \geq 1$. So $\psi_{n}(z)=\phi_{n}(z)$, for $n \in \mathbb{N}$.

(b) If $a_{1} \neq 0$ and $a_{2}=0$ then by (48) we get $\left|\psi_{2}(0)\right|=1$; which contradicts the quasi-definiteness of the linear functional associated with $\left\{\psi_{n}\right\}$.

(c) If $a_{1} a_{2} \neq 0$ then (50) gives $a_{n+1}=\frac{K_{n-1}}{K_{n}} a_{n}$; so $a_{n+1}=\frac{K_{1} a_{2}}{K_{n}}$, for $n \geq 2$. 
Now, because $a_{n} \neq 0$ for $n \geq 2$ we have from (49) $\psi_{n+1}(0)=0$ for $n \geq 2$. Applying the recurrence relation that $\left\{\psi_{n}\right\}$ satisfies we get

$$
\psi_{n+1}(z)=z \psi_{n}(z), n \geq 2 ;
$$

and so a representation for these polynomials

$$
\psi_{n}(z)=z^{n-2} \psi_{2}(z)
$$

follows. Hence, in order to determine $\left\{\phi_{n}\right\}$ we need $a_{1}, a_{2}, \phi_{1}(0)$ as initial conditions because $\phi_{2}(0)$ is defined by (48).

\section{SOME EXAMPLES.}

1. If $\phi_{1}(0)=0$ we have from (48)

$$
a_{2}=\phi_{2}(0) \bar{a}_{1}+a_{1}
$$

then, we can consider two situations:

(a) $a_{1}=a_{2}$

In this case $\phi_{2}(0) \bar{a}_{1}=0$ and as a consequence $\phi_{2}(0)=0$. From $(49) \phi_{n}(0)=0$, for $n \geq 1$ and from (50) $a_{n}=a_{1}, n \geq 1$.

This situation means that

$$
\begin{aligned}
\phi_{n}(z) & =z^{n} \\
a_{n} & =a_{1}, n \geq 1
\end{aligned}
$$

i.e. $\left(\psi_{n}\right)$ take the form $\psi_{n}(z)=z^{n-1}\left(z+a_{1}\right), n \geq 1$ and is orthogonal with respect to the Poisson measure $\mathrm{d} \mu=\frac{\mathrm{d} \theta}{\left|\exp (i \theta)+a_{1}\right|^{2}},\left|a_{1}\right| \neq 1$.

(b) $a_{1} \neq a_{2}$

Then, taking conjugates in (53)

$$
\begin{aligned}
& a_{2}=\phi_{2}(0) \bar{a}_{1}+a_{1} \\
& \bar{a}_{2}=\bar{\phi}_{2}(0) a_{1}+\bar{a}_{1}
\end{aligned}
$$

and $a_{1}=\frac{a_{2}-\bar{a}_{2} \phi_{2}(0)}{1-\left|\phi_{2}(0)\right|^{2}}$.

In this way, we can deduce

$$
\phi_{n+1}(0)=a_{n+1} \phi_{n}(0)=-\frac{K_{1} a_{2}}{K_{n}} \phi_{n}(0)
$$

and, thus because $\prod_{\imath=1}^{n} K_{\imath}=\Delta_{n}$ we have,

$$
\phi_{n+1}(0)=\left(-K_{1} a_{2}\right)^{n} \frac{1}{\Delta_{n}}, n \geq 2 .
$$

Remark that $\phi_{2}(0) \neq 0$ because of, otherwise, $a_{1}=a_{2}$ in contradiction with our hypothesis. Then $\psi_{n}(z)=z^{n-2} \psi_{2}(z)$ with $\psi_{2}(0) \neq 0$.

If we take $\phi_{2}(0)=q$ and $a_{2}=1-q$ then $a_{1}=\frac{1-q}{1+q}$. By induction

$$
\begin{aligned}
a_{n} & =\frac{1+(n-3) q}{1+(n-2) q}, n \geq 2 \\
\phi_{n}(0) & =(-1)^{n} \frac{q}{1+(n-2) q}, n \geq 2 \text { and } \phi_{1}(0)=0 .
\end{aligned}
$$


2. If $\phi_{1}(0) \neq 0$, we will consider two cases:

(a) $\phi_{2}(0)=0$

From (49) this implies that $\phi_{n}(0)=0, n \geq 2$. Then, $\phi_{n}(z)=z^{n-1} \phi_{1}(z), n \geq 1$. Moreover, $a_{n}=a_{2}$ and from (48) $a_{2}\left(1-\left|\phi_{1}(0)\right|^{2}\right)=a_{2} \bar{a}_{1} \phi_{1}(0)+a_{1}$. Taking conjugates

$$
\bar{a}_{2}\left(1-\left|\phi_{1}(0)\right|^{2}\right)=\bar{a}_{2} a_{1} \overline{\phi_{1}(0)}+\bar{a}_{1}
$$

and thus

$$
a_{1}=\bar{a}_{2} \frac{\left(1-\left|\phi_{1}(0)\right|^{2}\right)\left(1-a_{2} \overline{\phi_{1}(0)}\right)}{1-\left|a_{2}\right|^{2}\left|\phi_{1}(0)\right|^{2}}
$$

Observe that in this situation

$$
\begin{aligned}
\psi_{n}(z) & =z^{n-1} \phi_{1}(z)+a_{n} z^{n-2} \phi_{1}(z) \\
& =z^{n-2} \phi_{1}(z)\left(z+a_{2}\right) \\
& =z^{n-2}\left(z+\phi_{1}(0)\right)\left(z+a_{2}\right), n \geq 2
\end{aligned}
$$

This situation corresponds to the case when $\left\{\psi_{n}\right\}$ is a MOPS with respect to the measure $\mathrm{d} \mu=\frac{\mathrm{d} \theta}{\left|\exp (i \theta)+\phi_{1}(0)\right|^{2}\left|\exp (i \theta)+a_{2}\right|^{2}},\left|a_{2}\right| \neq 1$.

(b) $\phi_{2}(0) \neq 0$

Then $\phi_{n}(0) \neq 0, n \in \mathbb{N}$. This seems to be the more interesting situation.

An algorithm can be deduced in order to compute all the reflection parameters. In fact, from (48) we have

$$
\phi_{2}(0)=\frac{a_{2}\left(1-\left|\phi_{1}(0)\right|^{2}\right)-a_{1}-a_{1} \bar{a}_{2} \phi_{1}(0)}{\bar{a}_{1}} .
$$

Now, using sucessivelly (49) and (50) we get

$$
\begin{aligned}
& a_{3}=\frac{a_{2}}{1-\left|\phi_{2}(0)\right|^{2}} \quad \longrightarrow \phi_{3}(0)=-a_{3} \phi_{2}(0) \\
& a_{n+1}=\frac{a_{2}}{\prod_{k=1}^{n}\left(1-\left|\phi_{k}(0)\right|^{2}\right)} \longrightarrow \phi_{n+1}(0)=(-1)^{n+1} \prod_{k=2}^{n} a_{k} \phi_{1}(0) \text { for } n \geq 1 .
\end{aligned}
$$

Again, we can consider the particular situation $\phi_{2}(0)=q$ and $a_{2}=1-q$. As before, we can deduce

$$
\begin{aligned}
a_{n} & =\frac{1+(n-3) q}{1+(n-2) q} \\
\phi_{n}(0) & =(-1)^{n} \frac{q}{1+(n-2) q}
\end{aligned}
$$

for $n \geq 2$; if we take $\phi_{1}(0)=\frac{-q}{1-q}$ in (48) then $a_{1}=\frac{1-2 q}{1-q}$. Hence

$$
\begin{aligned}
a_{n} & =\frac{1+(n-3) q}{1+(n-2) q} \\
\phi_{n}(0) & =(-1)^{n} \frac{q}{1+(n-2) q}
\end{aligned}
$$

for $n \geq 1$.

ACKNOWLEDGEMENTS. This work was finished during a stay of the first author in Departamento de Ingeniería, Universidad Carlos III de Madrid, with the support of a grant from Junta Nacional de Investigação Científica e Tecnológica (JNICT) BD/2654/93/RM and Centro de Matemática da Universidade de Coimbra (CMUC). The work of the second author was supported by an Acción Integrada Hispano-Austriaca $94-12$ B.

The authors thank to the Professor Mourad Ismail for the suggestions and references that he gave to us. 


\section{References}

[1] Al-Salam, A.Verma. Some sets of orthogonal polynomials. Rev. Téc. Univ. Zulia. 9(2) (1986).

[2] A.Branquinho. Polinómıos ortogonais e funcıonaıs de momentos: Problemas ınversos. Master Thesis. Universidade de Coimbra. Coimbra 1993. (In Portuguese).

[3] A.Branquinho, F.Marcellán, J.Petronilho. Classical orthogonal polynomials: A functional approach. Acta Appl. Math. 34(3) (1994). 283-303.

[4] T.S.Chihara. On co-recursive orthogonal polynomials. Proc. Amer. Math. Soc. 8.(1957). 899-905.

[5] T.S.ChinaRA. An Introduction to Orthogonal Polynomials. Gordon and Breach. New York 1978.

[6] Ya.L.Geronimus. On polynomials orthogonal with respect to numerical sequences and on Hahn's theorem. Izv. Akad. Nauk. 4. (1940). 215-228. (In Russian).

[7] YA.L.Geronimus. On some finite-difference equations and the corresponding systems of orthogonal polynomials. Har'kov. Gos. Univ. Ucěn. Zap. 80 = Zap. Mat. Otd. Fiz.-Mat. Fak. i Har'kov. Mat. Obsec. (4) 25. (1957). 87-100. (In Russian).

[8] YA.L.Geronimus. Polynomials orthogonal on a circle and their applications. Amer. Math. Soc. Transl. Series 1. Vol. 3. Providence, RI (1962) 1-78.

[9] Z.S.GrinshPUn. On a class of orthogonal polynomials. Vest. Leningrad. Univ. Seria Mat. Mekha. i Astro. 21. (1966). 147-149. (In Russian).

[10] Z.S.Grinshpun. Differential equations for the Bernshtein-Szegö orthogonal polynomials. Diff. Eq. 26 (5). (1990). 545-550.

[11] F.Marcellán, P.Maroni. Orthogonal Polynomials on the Unit Circle and Their Derivatives. Constr. Approx. 7.(1991). 341-348.

[12] P.Maroni. Le Calcul des Formes Linéaires et les Polynômes Orthogonaux Semiclassiques. In "Orthogonal Polynomials and Their Applications". M.ALFARO et al. Eds. Lecture Notes in Mathematics 1329. Springer Verlag. Berlin. (1988). 279-290.

[13] P.Maroni. Sur la suite de polynômes orthogonaux associée à la forme $u=\delta_{c}+\lambda(x-c)^{-1} L$. Period.Math.Hung.21(3).(1990). 223-248.

[14] P.MARONI. Une théorie algébrique des polynômes orthogonaux. Application aux polynômes orthogonaux semiclassiques. In "Orthogonal Polynomials and their Applications". C.BREZINSKI, L.GORI and A.RONVEAUX Eds. J.C.Baltzer AG. Basel IMACS Annals on Computing and Applied Mathematics. 9 (1-4).(1991).95-130.

[15] A.F.Nikiforov, V.B.Uvarov. Special Functions of Mathematical Physics: An unified Approach. Birkhaüser Verlag. Basel. 1988.

[16] J.A.Shонат. On mechanical quadratures, in particular, with positive coefficients. Trans. Amer. Math. Soc. 42.(1937).461-496.

[17] G.Szegö. Orthogonal Polynomials. Amer.Math.Soc.Colloq.Publ.vol.23. Providence, Rhode Island 1975. (Fourth Edition).

[18] V.B.Uvarov. The connection between systems of polynomials orthogonal with respect to different distribution functions. USSR Compt. Math. Phys. (6) 9. (1969). 33-36.

[19] Y.Xu. A characterization of positive quadrature formulae. Math. Compt. (62) 206: (1994). 703-718. 


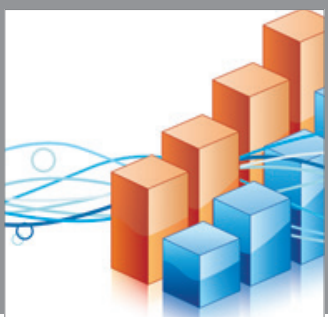

Advances in

Operations Research

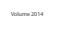

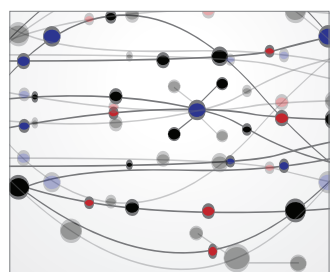

\section{The Scientific} World Journal
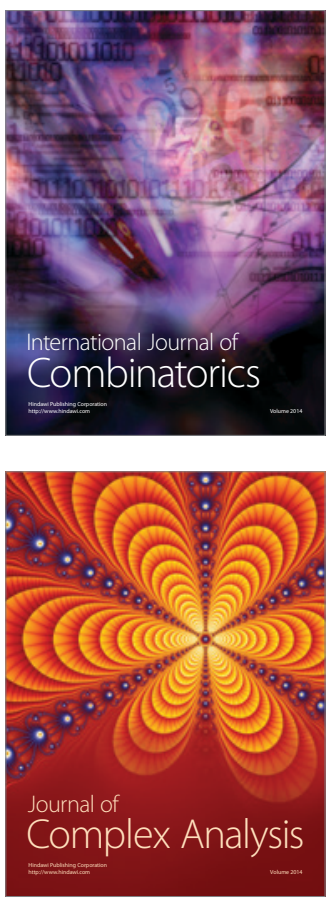

International Journal of

Mathematics and

Mathematical

Sciences
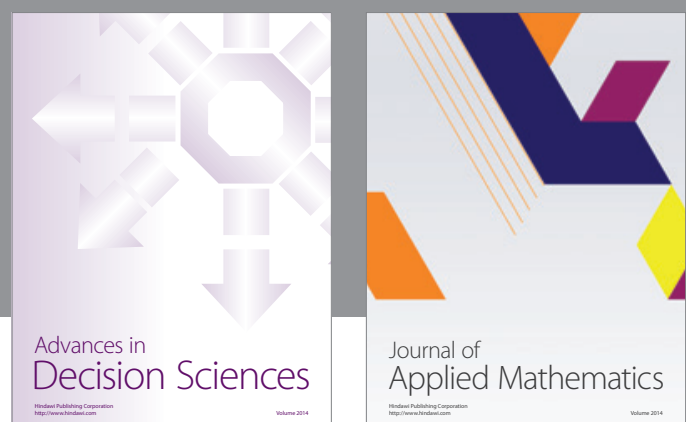

Journal of

Applied Mathematics
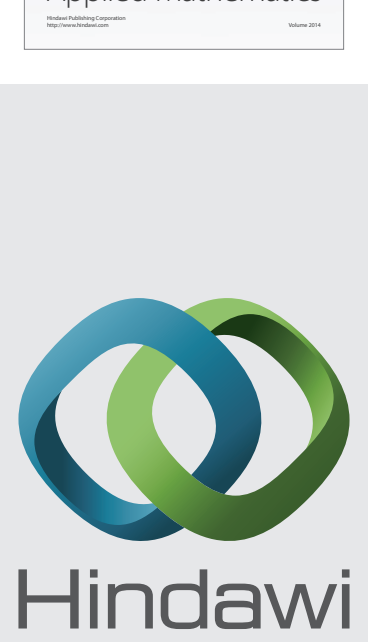

Submit your manuscripts at http://www.hindawi.com
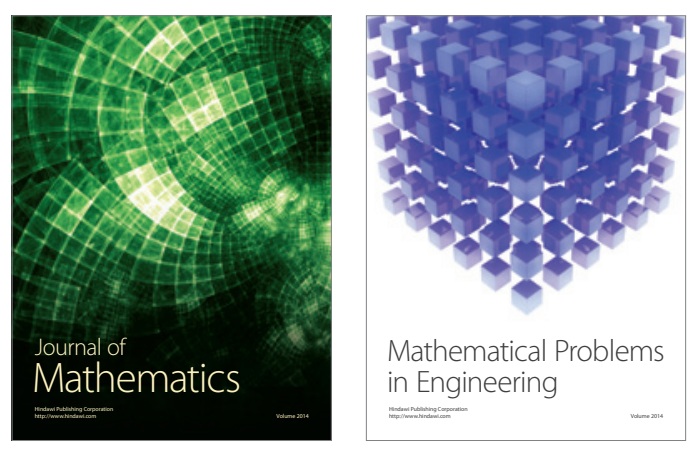

Mathematical Problems in Engineering
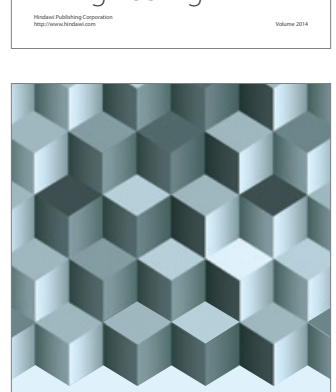

Journal of

Function Spaces
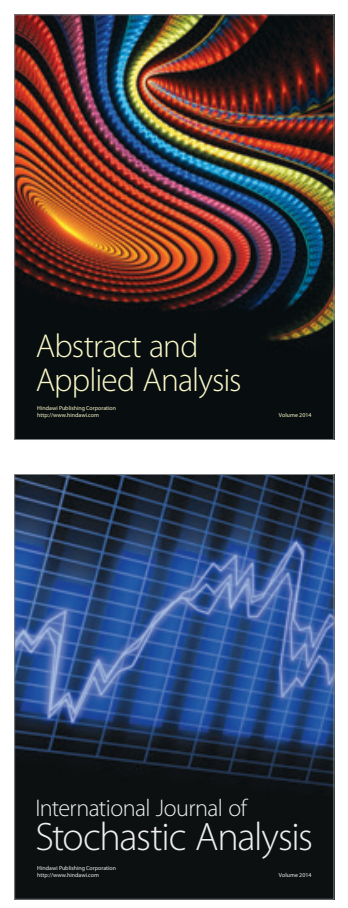

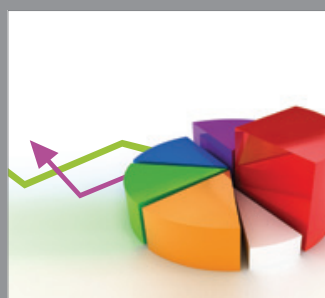

ournal of

Probability and Statistics

Promensencen
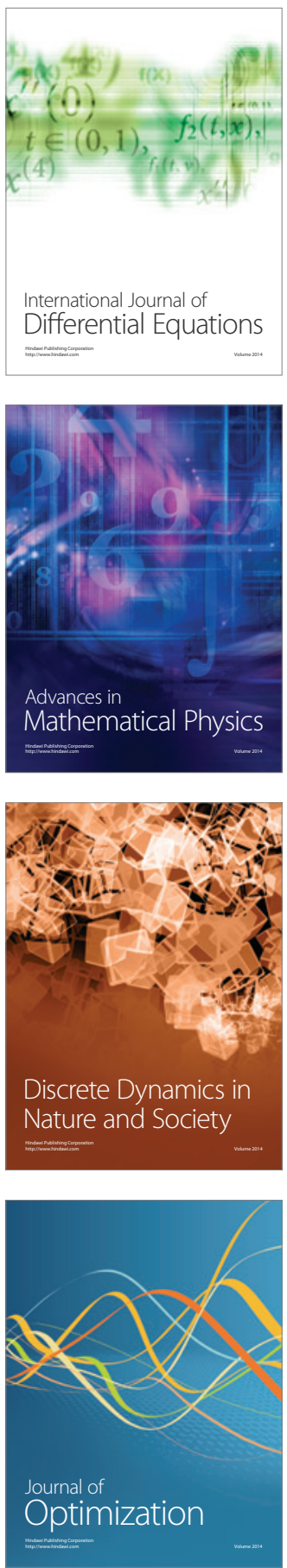